\title{
Therapeutic Role of Zinc Supplementation in Children Hospitalized with Pneumonia
}

\author{
Ghulam Shabbir Laghari ${ }^{1}$, Zahid Hussain ${ }^{2}$, Muhammad Taimur ${ }^{3}$, Nasir Jamil ${ }^{4}$ \\ 1. Pediatrics, Liaquat University of Medical and Health Sciences, Jamshoro, PAK 2. Pediatrics, National \\ Institute of Child Health, Karachi, PAK 3. Internal Medicine, Dow University of Health Sciences, Karachi, \\ PAK 4. Miscellaneous, University of Karachi, Karachi, PAK
}

$\square$ Corresponding author: Zahid Hussain, zahidhussain91393@gmail.com

Disclosures can be found in Additional Information at the end of the article

\section{Abstract}

\section{Introduction}

Pneumonia is one of the major causes of death in children younger than age five, especially in developing countries. The World Health Organization recommends children from a developing country take zinc supplements. We conducted this study to explore the efficacy of zinc supplementation in alleviating symptoms and shortening of hospital stay in children with pneumonia.

\section{Materials and methods}

We conducted this prospective, randomized controlled trial in the Department of Pediatrics, Civil Hospital, Jamshoro. We included 100 children of both genders randomized into two equal groups of zinc-supplemented and non-zinc-supplemented study groups after informed consent was obtained from the parents and legal guardians. The participants were aged 28 days to five years and admitted in the hospital with pneumonia. We monitored for hypoxia, tachypnea, chest indrawing, and cyanosis, and we recorded the total length of hospital stay for each group.

\section{Results}

We found no significant difference in symptom changes (i.e., hypoxia, tachypnea, chest indrawing, and cyanosis) between the zinc and non-zinc groups. However, hospital length of stay was significantly shorter for patients in the zinc-supplemented group compared to the non-zinc-supplemented group.

\section{Conclusion}

Received 03/22/2019

Review began 03/23/2019

Review ended 03/29/2019

Published 04/16/2019

\section{(c) Copyright 2019}

Laghari et al. This is an open access article distributed under the terms of the Creative Commons Attribution License CC-BY 3.0., which permits unrestricted use, distribution, and reproduction in any medium, provided the original author and source are credited.
Zinc supplementation did not yield a statistically significant reduction in symptoms in children with severe pneumonia. Zinc supplements given during an acute episode are not beneficial in short-term clinical recovery from severe pneumonia in hospitalized children.

Categories: Internal Medicine, Pediatrics, Pulmonology

Keywords: zinc, pneumonia, acute respiratory illness, pakistan, paediatrics

\section{Introduction}

Acute respiratory infections (ARIs) are the main cause of morbidity worldwide, and among ARIs, pneumonia is one of the major causes of death in children younger than age five, especially in 
developing countries. The increased risk for ARIs in developing countries can be linked to poverty, lower education levels, low birth weight, malnutrition, and lack of breastfeeding [1]. According to a 2013 National Demographics and Health Survey of Pakistan, 16\% of children under age five exhibited symptoms of ARI in the two weeks preceding the survey. These symptoms were more common in children younger than age two as compared to those aged from two to five years ( $20 \%$ vs. $13 \%$, respectively). Furthermore, pneumonia was determined to be the cause of death in around 91,000 children [2]. The World Health Organization and the United Nations Children's Fund recommend that children in developing countries should intake zinc supplements for 10 to 12 days as follows: $10 \mathrm{mg}$ daily for infants younger than six months and $20 \mathrm{mg}$ daily for infants older than six months to hasten recovery from severe pneumonia in developing countries. This can be linked to boosted immunity in response to zinc supplementation [3]. Zinc strengthens the immune system via its role in the maintenance of epithelial and tissue structure by promoting cell growth and reducing apoptosis. It also has antioxidant properties which protect against free radical damage sustained during inflammatory responses [4].

Various studies have highlighted the role of zinc in the management of severe pediatric pneumonia. Brooks et al. suggested that zinc supplemented in children younger than age two suffering from severe pneumonia resulted in a significant reduction in the severity of symptoms including tachypnea, anorexia, and restlessness; it also shortened the duration of hospital stay [5]. An Indian study showed that, compared to standard treatment, adding zinc to the treatment regime in children admitted with severe pneumonia resulted in faster improvement of symptoms and shortened duration of hospitalization [6]. However, there is still not enough local data to establish the efficacy of zinc in alleviating pneumonia symptoms in Pakistan.

The present study was conducted to determine the efficacy of zinc supplementation in alleviating symptoms and shortening of hospital stay in pediatric pneumonia.

\section{Materials And Methods}

We conducted a prospective, randomized controlled trial conducted in the Department of Pediatrics, Civil Hospital, Jamshoro. The study included children of both genders, aged 28 days to five years who were admitted to the hospital with pneumonia after informed consent from the parents and legal guardians.

A physical examination was performed to assess nasal flaring, respiratory rate per minute, breathing effort for the presence of chest indrawing, and peripheral cyanosis. The chest was auscultated for crepitations. Oxygen saturation was measured via pulse oximeter (hypoxia was taken as oxygen saturation $<92 \%$ ). Children with respiratory rate higher than the normal range for their age [7], with or without cough, and crepitations on chest auscultation were diagnosed with pneumonia, and children with a minimum of one danger sign (i.e., chest indrawing, cyanosis, grunting, and an inability to feed) were categorized as "severe pneumonia." Children with no crepitations or any other finding on chest auscultation were excluded. Children whose parents/guardians did not consent to the trial after brief detailing were also excluded. A total of 100 children were randomized into two groups using a random number generator: one group receiving $20 \mathrm{mg}$ /day oral zinc supplements, the other receiving no zinc supplements or placebo. Both groups were managed with standard pneumonia protocol including antimicrobial therapy, respiratory support, and fluid resuscitation.

Gender, age in months, and previous hospital admission due to respiratory illness in the previous month were recorded. The respiratory rate, arterial oxygen saturation levels, and signs of respiratory distress (i.e., nasal flaring, chest indrawing, cyanosis, inability to feed, and tachypnea) were noted at the time of admission and 48 hours of intervention in both groups. 


\section{Cureus}

The duration of the hospital stay was noted for every child. Data were entered and analyzed using IBM Statistical Package for the Social Sciences (SPSS) Statistics for Windows, version 21.0. (IBM Corp., Armonk, NY, US). Mean and standard deviation were calculated for continuous variables including age and respiratory rate. Categorized variables were presented as frequencies and percentages. The comparison was done within both groups at admission and 48 hours after admission. Dependent T-test was applied to compare means, and chi-square was applied to compare percentages. P-values of $\leqslant 0.05$ were considered statistically significant.

\section{Results}

There were 50 children in each group. The zinc group had $60 \%(n=30)$ male children, and the non-zinc group had $66 \%(n=33)$ male children. The mean age of the entire sample was $29 \pm 7$ months. Age, gender, and previous hospital admission due to respiratory illness in the last month for both study groups are shown in Table 1.

\section{Patient characteristics}

Gender

Male

Female

Age in months (mean $\pm \mathrm{SD})$

Previous hospital admission due to respiratory illness in the last one month

No episode

1-2 episodes

$>2$ episodes

\section{Zinc group Non-zinc group}

$\begin{array}{ll}30(60 \%) & 33(66 \%) \\ 20(40 \%) & 17(34 \%) \\ 27 \pm 6 & 30 \pm 4\end{array}$

$27(54 \%)$

$18(36 \%)$

$11(22 \%)$

$5(10 \%)$

\section{TABLE 1: Patient demographic characteristics at the time of admission}

SD: Standard deviation.

For both study groups, respiratory rate, oxygen saturation, and clinical signs of respiratory distress were recorded at the time of admission and at 48 hours of intervention. The mean respiratory rate, frequency of hypoxia, and frequency of signs of respiratory distress significantly improved after 48 hours of intervention in both the zinc group and non-zinc group as shown in Table 2. The mean duration of hospital stay was significantly shorter in the zincsupplemented group than the non-zinc group (Table 3). 


\section{Cureus}

\begin{tabular}{|c|c|c|c|c|c|c|}
\hline \multirow{2}{*}{ Patient characteristics } & \multicolumn{3}{|c|}{ Zinc group $(n=50)$} & \multicolumn{3}{|c|}{ Non-zinc group $(n=50)$} \\
\hline & At admission & At 48 hours & $\mathrm{p}$-value & At admission & At 48 hours & p-value \\
\hline Respiratory rate (mean \pm SD) & $49.69 \pm 6.27$ & $\begin{array}{l}39.87 \pm \\
4.72\end{array}$ & $<0.0001$ & $51.05 \pm 4.47$ & $45.28 \pm 2.58$ & $<0.0001$ \\
\hline Hypoxia (arterial $\mathrm{O}_{2}$ saturation $<92 \%$ ) & $16(32 \%)$ & $3(6 \%)$ & 0.001 & $18(36 \%)$ & $10(20 \%)$ & 0.07 \\
\hline \multicolumn{7}{|l|}{ Signs of respiratory distress } \\
\hline Nasal flaring & $22(44 \%)$ & $5(10 \%)$ & 0.0001 & $25(50 \%)$ & $9(18 \%)$ & 0.0007 \\
\hline Chest indrawing & $21(42 \%)$ & $7(14 \%)$ & 0.0001 & $23(46 \%)$ & $10(20 \%)$ & 0.005 \\
\hline Cyanosis & $2(4 \%)$ & NA & NA & $2(4 \%)$ & NA & NA \\
\hline Inability to feed & $8(16 \%)$ & NA & NA & $10(20 \%)$ & $2(4 \%)$ & 0.01 \\
\hline Tachypnea & $39(78 \%)$ & $13(26 \%)$ & $<0.00001$ & 42 (84\%) & 19 (38\%) & $<0.00001$ \\
\hline
\end{tabular}

\section{TABLE 2: Change in patient characteristics from admission to 48 hours in zinc and non-zinc group}

SD: Standard deviation; NA: Not applicable.

\section{TABLE 3: Mean duration of hospital stay in zinc and non-zinc group}

SD: Standard deviation.

\section{Discussion}

Both study groups demonstrated improvements in respiratory rate, oxygen saturation, and respiratory distress after 48 hours of intervention. The zinc-supplemented group had a shorter mean duration of hospital stay compared to the non-zinc-supplemented group. Our findings were similar to those of a meta-analysis of seven randomized controlled trials in which the therapeutic role of zinc with standard therapy was compared with standard therapy alone in 1,066 children from developing countries admitted for acute lower respiratory tract infections. There was no significant difference in the resolution of severe illness measured using symptoms such as hypoxia, chest indrawing, and tachypnea [8].

Das et al. found no significant difference between zinc and non-zinc groups in terms of 
hospital stay, while in our study, hospital stay was significantly shorter in the zinc group compared to the non-zinc group [8]. Even when compared to neighboring countries such as Bangladesh, India, and Nepal, a systemic review of four articles in 2011 with 3,200 children failed to prove a significant effect on the clinical recovery of pneumonia when zinc is added to the treatment regime [9]. In 2016, another meta-analysis of nine studies failed to established zinc as an adjunct therapy to antibiotics in children suffering from pneumonia. There was no significant reduction in time to recovery, hospital length of stay, treatment failure, and change of antibiotic when compared to placebo [10]. Therefore, our study, compared with other metaanalyses and systemic reviews, suggests there is no therapeutic role of zinc in children hospitalized with pneumonia.

However, Brooks et al. [5] and Coles et al. [6] reported a significant improvement in symptoms in zinc-supplemented participants as compared to placebo. A review of six randomized doubleblind and placebo-controlled trials of adjunct zinc therapy in patients with severe pneumonia failed to show significantly improved clinical outcomes; however, a significant reduction in mortality due to severe pneumonia was reported for the zinc-supplemented groups [11]. In another study, the zinc-supplemented group experienced reduced time for symptom resolution and had shorter hospital stay durations compared to patients receiving a placebo [12].

The evidence regarding the role of zinc as an adjunct therapy in children with severe pneumonia is controversial. As discussed above, where some major clinical trials have cast zinc in a promising light, others have shown no clinical benefit to patients. While this study reported robust results, it was not without certain limitations. The age of participants in this study was very diverse (from age 28 days to five years), and different ages have various presentations, severities, and outcomes for pneumonia. Younger children have more severe pneumonia than older children. Also, this study included clinical parameters but not serological characteristics of the children. This study did not report patient outcomes in terms of mortality and discharge. Future clinical studies should be designed to evaluate the role of zinc in severe pneumonia with the addition of assessing clinical, radiological, and serological parameters, and measure disease outcome in terms of the resolution of symptoms, duration of hospital stay, and mortality.

\section{Conclusions}

Zinc supplementation provided no statistically significant reduction in symptoms of severe pneumonia in children under age five. Zinc supplementation given during an acute episode does not help in the short-term clinical recovery from severe pneumonia in hospitalized children.

\section{Additional Information Disclosures}

Human subjects: Consent was obtained by all participants in this study. Liaquat University of Medical and Health Sciences, Jamshoro issued approval 18-701. I am pleased to inform you that the chairman, on behalf of the committee members of LUMHS Jamshoro, approves ethical clearance of the above-mentioned research project on this 6th day of October 2018. The favorable opinion is given for a period of 1 year from the date of this letter and the validity may be renewed for a further period on a fresh application. Animal subjects: All authors have confirmed that this study did not involve animal subjects or tissue. Conflicts of interest: In compliance with the ICMJE uniform disclosure form, all authors declare the following: Payment/services info: All authors have declared that no financial support was received from any organization for the submitted work. Financial relationships: All authors have declared that they have no financial relationships at present or within the previous three years with any organizations that might have an interest in the submitted work. Other relationships: All 
authors have declared that there are no other relationships or activities that could appear to have influenced the submitted work.

\section{References}

1. Cashat-Cruz M, Morales-Aguirre JJ, Mendoza-Azpiri M: Respiratory tract infections in children in developing countries. Semin Pediatr Infect Dis. 2005, 16:84-92. 10.1053/j.spid.2005.12.005

2. Pakistan demographics and health survey, 2012-13 . (2013). Accessed: March 21, 2019: https://www.nips.org.pk/abstract_files/PDHS Final Report as of Jan 22-2014.pdf .

3. Kliegman RM, Stanton BF, Geme JWS, Schor NF, Behrman RE: Nelson Textbook of Pediatrics. Elsevier, Philadelphia; 2016.

4. Berger A: What does zinc do?. BMJ. 2002, 325:1062. 10.1136/bmj.325.7372.1062

5. Brooks WA, Yunus M, Santosham M, Wahed MA, Nahar K, Yeasmin S, Black RE: Zinc for severe pneumonia in very young children: double-blind placebo-controlled trial. Lancet. 2004, 363:1683-1688. 10.1016/S0140-6736(04)16252-1

6. Coles CL, Bose A, Moses PD, Mathew L, Agarwal I, Mammen T, Santosham M: Infectious etiology modifies the treatment effect of zinc in severe pneumonia. Am J Clin Nutr. 2007, 86:397-403. 10.1093/ajcn/86.2.397

7. Normal values in children . (2018). Accessed: March 21, 2019: https://www.aclsmedicaltraining.com/normal-values-in-children/.

8. Das RR, Singh M, Shafiq N: Short-term therapeutic role of zinc in children $<5$ years of age hospitalised for severe acute lower respiratory tract infection. Paediatr Respir Rev. 2012, 13:184-191. 10.1016/j.prrv.2012.01.004

9. Haider BA, Lassi ZS, Ahmed A, Bhutta ZA: Zinc supplementation as an adjunct to antibiotics in the treatment of pneumonia in children 2 to 59 months of age. Cochrane Database Syst Rev. 2011, 10:CD007368. 10.1002/14651858.CD007368.pub2

10. Tie HT, Tan Q, Luo MZ, Li Q, Yu JL, Wu QC: Zinc as an adjunct to antibiotics for the treatment of severe pneumonia in children $<5$ years: a meta-analysis of randomisedcontrolled trials. Br J Nutr. 2016, 115:807-816. 10.1017/S0007114515005449

11. Wang L, Song Y: Efficacy of zinc given as an adjunct to the treatment of severe pneumonia: a meta-analysis of randomized, double-blind and placebo-controlled trials. Clin Respir J. 2018, 12:857-864. 10.1111/crj.12646

12. Valavi E, Hakimzadeh M, Shamsizadeh A, Aminzadeh M, Alghasi A: The efficacy of zinc supplementation on outcome of children with severe pneumonia. A randomized double-blind placebo-controlled clinical trial. Indian J Pediatr. 2011, 78:1079-1084. 10.1007/s12098-0110458-1 